\title{
Erlotinib has better efficacy than gefitinib in adenocarcinoma patients without $E G F R$-activating mutations, but similar efficacy in patients with $E G F R$-activating mutations
}

\author{
WEN-SHUO WU ${ }^{1}$, YUH-MIN CHEN ${ }^{1,2}$, CHUN-MING TSAI $^{1}$, JEN-FU SHIH $^{1}$, CHAO-HUA CHIU ${ }^{1}$, \\ KUN-TA CHOU $^{1}$, SHINN-LIANG LAI ${ }^{1}$, CHIEH-HUNG WU ${ }^{1}$, YUNG-HUNG LUO ${ }^{1}$, CHU-YUN HUANG ${ }^{1}$, \\ YU-CHIN LEE ${ }^{1}$, REURY-PERNG PERNG ${ }^{1}$ and JACQUELINE WHANG-PENG ${ }^{2}$ \\ ${ }^{1}$ Chest Department, Taipei Veterans General Hospital, School of Medicine, \\ National Yang-Ming University; ${ }^{2}$ CECR, School of Medicine, Taipei Medical University, Taipei, Taiwan, R.O.C.
}

Received August 23, 2011; Accepted October 4, 2011

DOI: 10.3892/etm.2011.383

\begin{abstract}
Epidermal growth factor receptor-tyrosine kinase inhibitors (EGFR-TKIs) are an effective treatment for advanced non-small cell lung cancer. The objective of the present study was to compare the efficacy of gefitinib and erlotinib in patients with pulmonary adenocarcinoma, whose tumor EGFR mutation status was known. Pulmonary adenocarcinoma patients who began receiving gefitinib or erlotinib treatment from January 2005 to December 2010, and whose tumor EGFR mutation status had been determined, were included. Clinical data, type of treatment response and survival time data were collected. Of the 224 patients enrolled, 124 received gefitinib treatment and 100 received erlotinib treatment. Of these patients, 146 individuals had tumors with EGFR-activating mutations (exon 19 deletions and/point mutation of L858R in exon 21) and 78 did not. There was no difference in treatment response whether or not the patients had tumors with EGFR-activating mutations at the time they received gefitinib or erlotinib treatment. The median progression-free survival (PFS) of the gefitinib and erlotinib groups was 7.6 and 7.9 months, respectively $(\mathrm{p}=0.4731)$. PFS was significantly longer for patients without EGFR-activating mutations who received erlotinib treatment $(n=48$; median, 4.5 months) than for those who received gefitinib treatment ( $\mathrm{n}=30$; median, 2.3 months), with a hazard ratio of 0.58 (95\% CI, 0.35-0.96; $\mathrm{p}=0.0339$ ). Patients whose tumors had EGFR-activating mutations displayed no difference in PFS with either gefitinib ( $\mathrm{n}=94$; median, 10.5 months) or erlotinib treatment ( $\mathrm{n}=52$; median, 10.4 months). In conclusion, PFS
\end{abstract}

Correspondence to: Dr Yuh-Min Chen, Chest Department, Taipei Veterans General Hospital, 201, Sec. 2, Shih-pai Road, Taipei 112, Taiwan, R.O.C.

E-mail: ymchen@vghtpe.gov.tw

Key words: epidermal growth factor receptor, tyrosine kinase inhibitors, non-small cell lung cancer showed no difference with either agent in patients whose tumors had EGFR-activating mutations, but was significantly longer in patients whose tumors did not have EGFR-activating mutations when receiving erlotinib treatment.

\section{Introduction}

Worldwide, lung cancer is the leading cause of cancer mortality (1). Major progression in understanding molecular cancer biology and in determining the mechanisms of oncogenesis during the last decade has resulted in the development of molecular targets for non-small cell lung cancer (NSCLC) treatments. Inhibition of the epidermal growth factor receptor (EGFR) pathway with tyrosine kinase inhibitors (TKIs), such as gefitinib and erlotinib, provides an effective and promising treatment for NSCLC, as either first-line or salvage therapy, with the added advantage of improved efficacy, tolerability and quality of life compared with other chemotherapy agents (2-9). It has been demonstrated that a subset of patients (female, never smoked, East Asian with an adenocarcinoma diagnosis) may respond better to EGFR-TKIs. A higher prevalence of sensitive EGFR-activating mutations (deletion in exon 19 or point mutation of L858R in exon 21) has been found in this subset of patients (10-12).

Gefitinib and erlotinib were each compared with a placebo in phase III randomized trials (ISEL and BR.21, respectively) in which the majority of enrolled patients were Caucasian $(2,3)$. The varying overall survival outcomes with these two drugs compared with the placebo were widely debated, although gefitinib demonstrated a significant survival benefit in a subgroup of patients of Asian origin (4). There are many possible reasons for this difference in survival, including the most frequently mentioned - that the dose intensity or drug concentrations are higher in patients receiving erlotinib treatment than in those receiving gefitinib treatment (13-17). Phase III, randomized trials comparing gefitinib or erlotinib with docetaxel or pemetrexed have been performed, but the superiority of one agent over the other has not been documented $(5,18,19)$. To our knowledge, there is no published prospective trial to date, which compares gefitinib and erlotinib treatment, not to mention a 
study performed based on the tumor EGFR mutation status. In the present study, we retrospectively evaluated the difference in efficacy between these two agents in Taiwanese patients with advanced adenocarcinoma, whose tumor EGFR mutation status was known. The aim of the study was to identify any difference in the efficacy of these agents in patients with or without EGFR-activating mutations.

\section{Methods and materials}

Patients. This study was approved by our Institutional Review Board (VGHIRB 2011-04-0151A). Between January 2005 and December 2010, patients who received the standard dose of gefitinib (250 mg daily) or erlotinib (150 mg daily) with assessable and inoperable stage IIIB or IV adenocarcinoma were enrolled into this retrospective study. Medical charts and images to evaluate treatment response were retrospectively reviewed. Clinical characteristics of the patients, including age, gender, Eastern Cooperative Oncology Group (ECOG) performance status (PS), tumor histological type, stage, smoking history and present EGFR-TKI therapy, were recorded. Patients were defined as non-smokers if they had smoked $<100$ cigarettes in their lifetime, or smokers if they had smoked $\geq 100$ cigarettes in their lifetime. The date that EGFR-TKI treatment was commenced, time to disease progression and date of death or last follow-up were also recorded.

Efficacy evaluation. Baseline assessments were performed within 3 weeks prior to EGFR-TKI treatment. A chest computed tomography scan (including liver and adrenal glands) was performed within 3 weeks prior to commencing EGFR-TKI treatment, every 2 to 3 months thereafter or when confirmation of treatment response or disease progression was required. Treatment response evaluation was performed according to the Response Evaluation Criteria in Solid Tumors (RECIST) group criteria (20). Progression-free survival (PFS) was calculated from the date of administration of the first dose of EGFR-TKI to the earliest sign of disease progression, as determined by RECIST (20), or death from any cause. If disease progression had not occurred at the time of the last follow-up visit, PFS was considered to have been censored at that time. Survival was measured from administration of the first dose of EGFR-TKI until the date of death or last follow-up.

EGFR mutation analysis. The EGFR mutation analysis was performed with nucleotide sequence analysis. The VarientSEQr ${ }^{\mathrm{TM}}$ Resequencing Primer Set [Applied Biosystems, Inc. (ABI), Foster City, CA, USA] was selected for mutational analysis of the tyrosine kinase domain, exons 18-21 of the EGFR gene. Genomic DNA was extracted from paraffin blocks, exons 18-21 were amplified and uncloned polymerase chain reaction (PCR) fragments were sequenced and analyzed in both sense and antisense directions for the presence of heterozygous mutations. Normal, control DNA provided by the ABI company was used for wild-type control. All sequence variations were confirmed by multiple, independent PCR amplifications and repeated sequencing reactions. EGFR-activating mutations were defined as those with exon 19 deletions or point mutation of L858R in exon 21.
Statistical analysis. All categorical variables were analyzed with Chi-square tests. Median PFS and overall survival were estimated using the Kaplan-Meier method and log-rank test. Hazard ratios (HRs) in the overall population and in patient subsets were calculated using the Cox proportional hazards model. All p-values were 2 -sided and a value of $<0.05$ was considered to be statistically significant. All statistical analyses were performed using SPSS software (SPSS Inc., Chicago, IL, USA).

\section{Results}

Patients. A total of 224 patients who received EGFR-TKI treatment and whose tumor EGFR mutation status was known were enrolled; of these, 124 received gefitinib treatment and 100 received erlotinib treatment. Since 2 to 4 weeks are required for the results of tumor EGFR mutation analysis to be available, a large proportion ( $>90 \%$ ) of the patients commenced gefitinib or erlotinib treatment before these results were known. Of the 224 patients, 146 had tumors with EGFR-activating mutations (96 exon 19 deletions alone, 41 L858R mutations, and 9 with both exon 19 deletions and L858R mutations) and 78 did not (15 atypical mutations in either exon 18, 20 and/or 21, and 63 wild-type).

Although more male patients received erlotinib treatment and more patients who never smoked received gefitinib treatment, there was no statistically significant difference in gender, smoking, performance and treatment response between the patients who received either treatment. However, more patients with EGFR-activating mutations received gefitinib than erlotinib $(\mathrm{p}<0.001$; Table I). With regard to the activating mutation incidence in relation to the clinical predictors commonly used, males had a $67.6 \%$ mutation rate and females had a $62.9 \%$ mutation rate $(p=0.486)$; non-smokers had a $66.7 \%$ mutation rate, while smokers had a $61.3 \%$ mutation rate $(\mathrm{p}=0.531)$. EGFR-TKI was the firstline treatment in 75 patients, second-line treatment in 126 patients, third-line treatment in 3 patients and fourth-line treatment in 1 patient.

Treatment response. There was no difference in the type of response between the 2 agents $(\mathrm{p}=0.141)$. Patients with the best partial response, stable disease, or progressive disease during treatment were the following: 52 (41.9\%), $46(37.1 \%)$ and $26(21 \%)$, respectively (among 124 patients who received gefitinib), and 42 (42\%), 27 (27\%) and 31 (31\%), respectively (among 100 patients who received erlotinib). The objective response rate to EGFR-TKI was similar between patients who received gefitinib or erlotinib (41.9 vs. $42 \%, \mathrm{p}=1.000$; Table II). When treatment response was classified according to tumor EGFR mutation status, 78 out of 146 patients $(53.4 \%)$ with EGFR-activating mutations had a partial response to EGFR-TKI treatment, while only 16 out of 78 patients $(20.5 \%)$ without EGFR-activating mutations responded to the treatment $(\mathrm{p}<0.001)$.

When the type of treatment response was classified according to the EGFR mutation status and EGFR-TKI treatment was used, there was no significant difference in patients with EGFR-activating mutations who received gefitinib or erlotinib. In addition, there was no significant difference 
Table I. Clinical characteristics of the 224 patients who received gefitinib or erlotinib treatment.

\begin{tabular}{|c|c|c|c|}
\hline \multirow[b]{2}{*}{ Variables } & \multicolumn{2}{|c|}{ Patient number (\%) } & \multirow[b]{2}{*}{ p-value ${ }^{a}$} \\
\hline & Gefitinib $(n=124)$ & Erlotinib $(\mathrm{n}=100)$ & \\
\hline Gender & & & 0.081 \\
\hline Male & $53(49.1)$ & $55(50.9)$ & \\
\hline Female & $71(61.2)$ & $45(38.8)$ & \\
\hline Smoking history & & & 0.071 \\
\hline No & $96(59.3)$ & $66(40.7)$ & \\
\hline Yes & $28(45.2)$ & $34(54.5)$ & \\
\hline Performance status & & & 0.43 \\
\hline 0 & $21(61.8)$ & $13(38.2)$ & \\
\hline 1 & $72(50.7)$ & $70(49.3)$ & \\
\hline 2 & $20(66.7)$ & $10(33.3)$ & \\
\hline 3 & $6(66.7)$ & $3(33.3)$ & \\
\hline 4 & $5(55.6)$ & $4(44.4)$ & \\
\hline Type of response to TKI & & & 0.141 \\
\hline Partial response & $52(55.3)$ & $42(44.7)$ & \\
\hline Stable disease & $46(63)$ & $27(37)$ & \\
\hline Progressive disease & $26(45.6)$ & $31(54.4)$ & \\
\hline EGFR-activating mutations & & & $<0.001$ \\
\hline With & $94(64.4)$ & $52(35.6)$ & \\
\hline Without & $30(38.5)$ & $48(61.5)$ & \\
\hline
\end{tabular}

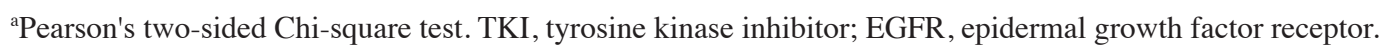

Table II. Type of treatment response and the relationship to EGFR mutation status

\begin{tabular}{|c|c|c|c|}
\hline & Gefitinib, no. $(\%)$ & Erlotinib, no. $(\%)$ & p-value ${ }^{a}$ \\
\hline With activating mutations & & & 0.277 \\
\hline Partial response & $48(51.1)$ & $30(57.7)$ & \\
\hline Stable disease & $35(37.2)$ & $13(25)$ & \\
\hline Progressive disease & $11(11.7)$ & $9(17.3)$ & \\
\hline Without activating mutations & & & 0.446 \\
\hline Partial response & $4(13.3)$ & $12(25)$ & $0.064^{\mathrm{b}}$ \\
\hline Stable disease & $11(36.7)$ & $14(29.2)$ & \\
\hline Progressive disease & $15(50)$ & $22(45.8)$ & \\
\hline
\end{tabular}

${ }^{a}$ Pearson's two-sided Chi-square test; ${ }^{b}$ when partial response or no partial response was compared between the two agents. EGFR, epidermal growth factor receptor.

in patients without EGFR-activating mutations, other than erlotinib, which had a numerically higher response rate than gefitinib among these patients, with borderline significance $(\mathrm{p}=0.064$; Table II).

PFS. There was no difference in PFS between the 124 patients treated with gefitinib (censor, 28) and the 100 treated with erlotinib (censor, 25) (median, 7.6 vs. 7.9 months; HR, 0.89; 95\% CI, 0.66-1.21; p=0.4731) (Fig. 1). PFS was significantly longer in the 146 patients (censor, 40) whose tumors had
EGFR-activating mutations than in the 78 patients (censor, 13) whose tumors did not have EGFR-activating mutations (median, 10.5 vs. 2.5 months; HR, 0.48; 95\% CI, 0.35-0.66; $\mathrm{p}<0.0001)$. There was no statistically significant difference in PFS between males and females, and between EGFR-TKI used as first-line or later lines of treatment. However, PFS was significantly different in patients with different staging statuses and varying PS (Table III).

The Cox-regression test for multivariate analysis of PFS, including EGFR mutation status (with or without activating 
Table III. PFS based on clinical characteristics and EGFR mutation status.

\begin{tabular}{|c|c|c|c|}
\hline & Median PFS, months & Hazard ratio $(95 \% \mathrm{CI})$ & $\mathrm{p}$-value $\mathrm{a}^{\mathrm{a}}$ \\
\hline Treatment & & $0.89(0.66-1.21)$ & 0.4731 \\
\hline Gefitinib, $n=124$ & 7.6 & & \\
\hline Erlotinib, $n=100$ & 7.9 & & \\
\hline EGFR-activating mutations & & $0.48(0.35-0.66)$ & $<0.0001$ \\
\hline No, $n=78$ & 2.5 & & \\
\hline Yes, $n=146$ & 10.5 & & \\
\hline Gender & & $0.75(0.56-1.03)$ & 0.0707 \\
\hline Male, $n=108$ & 9.8 & & \\
\hline Female, $n=116$ & 7.6 & & \\
\hline Staging & & $0.40(0.18-0.90)$ & 0.0222 \\
\hline IIIB, $n=13$ & 15.1 & & \\
\hline $\mathrm{IV}, \mathrm{n}=211$ & 7.6 & & \\
\hline Performance status & & $<0.0001$ & \\
\hline $0, \mathrm{n}=34$ & 7.4 & & \\
\hline $1, \mathrm{n}=142$ & 9.8 & $0.42(0.19-0.94)$ & \\
\hline $2, n=30$ & 4.6 & $0.51(0.25-1.04)$ & \\
\hline $3, \mathrm{n}=9$ & 1.3 & $0.81(0.36-1.78)$ & \\
\hline $4, n=9$ & 2.8 & $3.57(1.35-9.41)$ & \\
\hline TKI treatment as & & & 0.2917 \\
\hline First-line, $n=75$ & 7.4 & & \\
\hline Second-line, $n=126$ & 8 & $0.85(0.50-1.45)$ & \\
\hline Third-line, $\mathrm{n}=22$ & 4.6 & $1.08(0.65-1.79)$ & \\
\hline Fourth-line, $\mathrm{n}=1$ & $>19.4$ & & \\
\hline
\end{tabular}

${ }^{a}$ Kaplan-Meier analysis and log-rank test. PFS, progression-free survival; EGFR, epidermal growth factor receptor; TKI, tyrosine kinase inhibitor.

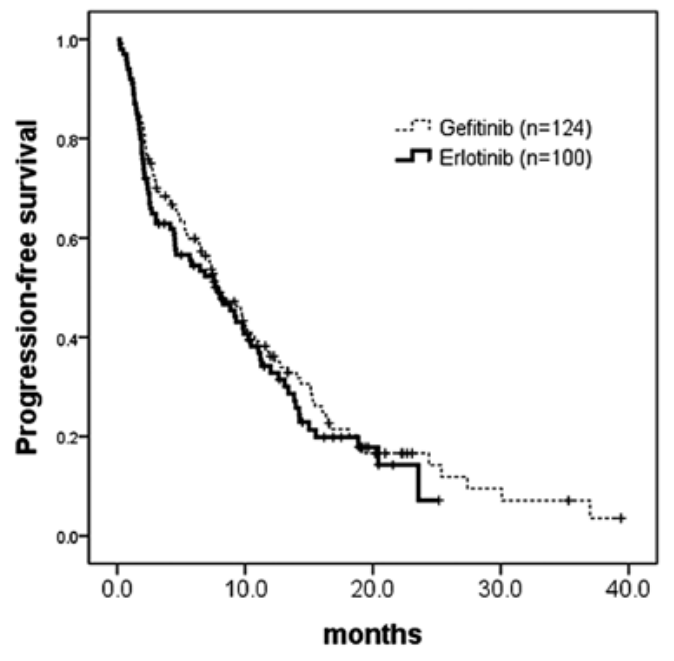

Figure 1. Kaplan-Meier analysis of progression-free survival (PFS) by treatment agents. PFS did not differ in the 124 gefitinib-treated patients (censor, 28; median, 7.6 months) compared to the 100 erlotinib-treated patients (censor, 25; median, 7.9 months) ( $\mathrm{p}=0.4731$; HR, 0.89; 95\% CI, 0.66-1.21). HR, hazard ratio.

mutations), treatment regimen (gefitinib or erlotinib), gender (male or female), PS (0, 1, 2, 3 or 4$)$, staging (IIIB or IV) and present line of treatment (first-, second-, third- or fourth-line), revealed that only tumors with EGFR-activating mutations (HR, 0.37; 95\% CI, 0.25-0.53; p<0.0001) and PS ( $<<0.0001)$ had a statistically significant effect on PFS.

When an EGFR-TKI regimen was administered to the patients whose tumors had EGFR-activating mutations, there was no statistical difference in PFS between the 94 patients (censor, 26) who received gefitinib and 52 (censor, 14) who received erlotinib (median, 10.5 vs. 10.3 months; HR, 1.22; 95\% CI, 0.82-1.83; p=0.3224; Fig. 2). When EGFR-TKI was administered to the patients whose tumors did not have EGFR-activating mutations, the 48 (censor, 11) who received erlotinib had a significantly longer PFS than the 30 (censor, 2) who received gefitinib (median, 4.5 vs. 2.3 months; HR, 0.58; 95\% CI, 0.35-0.96; p=0.0315; Fig. 3).

Overall survival analysis revealed no survival difference in patients with EGFR-activating mutations who received either gefitinib ( $n=94$; censor, 54; median, 26 months) or erlotinib treatment $(\mathrm{n}=52$; censor, 40; median, not reached) (HR, 0.49; 95\% CI, 0.36-1.22; p=0.2728; Fig. 4A). There was also no survival difference in patients without EGFR-activating mutations who received either gefitinib treatment $(n=30$; censor, 14; median, 8.6 months) or erlotinib treatment ( $\mathrm{n}=48$; censor, 31 ; median, not reached) (HR, 0.58; 95\% CI, 0.26-1.14; $\mathrm{p}=0.1104$; 


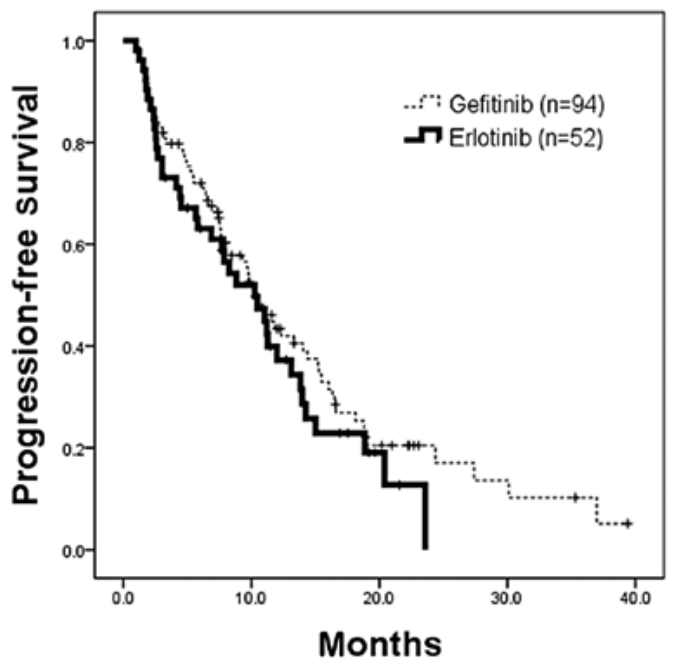

Figure 2. Kaplan-Meier analysis of progression-free survival (PFS) in patients whose tumors had EGFR-activating mutations who received gefitinib or erlotinib. There was no statistical difference in PFS of 94 patients who received gefitinib (censor, 26; median, 10.5 months) and 52 patients who received erlotinib (censor, 14; median, 10.3 months) ( $\mathrm{p}=0.3224$; HR, 1.22; 95\% CI, 0.82-1.83). EGFR, epidermal growth factor receptor; HR, hazard ratio.

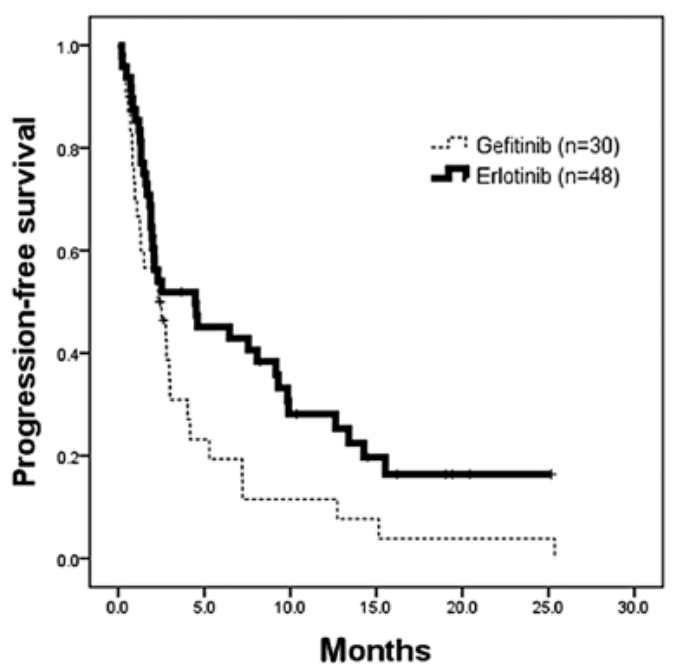

Figure 3. Kaplan-Meier analysis of progression-free survival (PFS) in patients whose tumors did not have EGFR-activating mutations who received gefitinib or erlotinib. There was significant longer PFS for the 48 patients who received erlotinib (censor, 11; median, 4.5 months) than the 30 patients who received gefitinib (censor, 2; median, 2.3 months) $(\mathrm{p}=0.0315$; HR, 0.58; 95\% CI, 0.35-0.96). HR, hazard ratio.

Fig. 4B). Overall survival could not be interpreted due to the inadequate number of deaths.

\section{Discussion}

Treatment with one of the EGFR-TKIs, gefitinib or erlotinib, has become an important option for patients with advanced NSCLC. The tumor EGFR mutation rate is approximately 3 times more prevalent in Asian patients than in Caucasians. An effect on overall survival in genotypically uncharacterized NSCLC patients was observed with erlotinib (BR.21 trial) (3), but not gefitinib (ISEL trial) (2), although the response rates
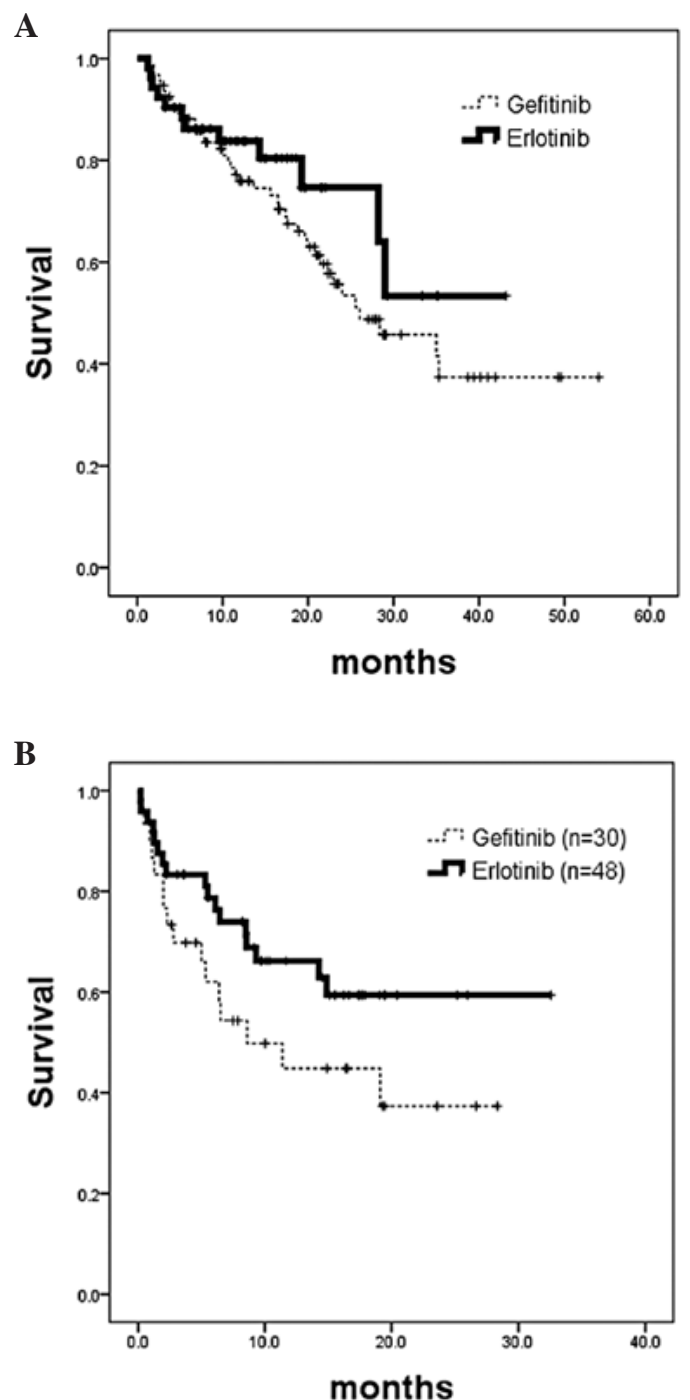

Figure 4. Kaplan-Meier analysis of overall survival calculated from patients commencing EGFR-TKI treatment. (A) Patients whose tumors had EGFR-activating mutations. No survival difference was noted in patients who received gefitinib treatment ( $\mathrm{n}=94$; censor, 54; median, 26 months) or erlotinib treatment $(\mathrm{n}=52$; censor, 40 ; median, not reached) $(\mathrm{p}=0.2728$; HR, 0.49; 95\% CI, 0.36-1.22). (B) Patients whose tumors did not have EGFR-activating mutations. No survival difference was noted in patients who received gefitinib treatment ( $\mathrm{n}=30$; censor, 14; median, 8.6 months) or erlotinib treatment ( $\mathrm{n}=48$; censor, 31 ; median, not reached) $(\mathrm{p}=0.1104$; HR, 0.58; 95\% CI, 0.29-1.14). TKI, tyrosine kinase inhibitor; EGFR, epidermal growth factor receptor; HR, hazard ratio.

were similar. Several possible explanations have been offered, and the focus has mainly been on the suboptimal dosage of gefitinib (17). Erlotinib was administered at its maximum tolerated dose (MTD), while gefitinib was administered at approximately one third of its MTD (13-16). Erlotinib treatment may be more efficacious than gefitinib in EGFR-wild type or atypical mutated lung tumors, since erlotinib inhibits the activity of wild-type EGFR-TKI in tumor cells at 50\% inhibitory concentrations of 2-20 nmol/l. By contrast, several-fold higher drug concentrations of gefitinib are required to block wild-type or atypical mutated EGFR signaling (21-24). This result was supported by evidence from patients lacking EGFR-activating mutations who still achieved a benefit from erlotinib following failure of gefitinib treatment (25). By contrast, for patients or cell-lines 
with EGFR-activating mutations, such as exon 19 deletions and exon 21 L858R point mutations, both gefitinib and erlotinib are highly effective $(26,27)$. Clinical data from a recent molecular analysis of the BR.21 trial also demonstrated that the survival impact of erlotinib was not confounded significantly by tumor cell EGFR mutation status (28). Furthermore, the SATURN trial, which tested maintenance erlotinib following chemotherapy revealed that PFS and overall survival were prolonged in patients with or without EGFR-activating mutations (29). These results suggest that erlotinib treatment is beneficial, irrespective of EGFR mutation status. The present study revealed a better response rate and PFS in patients whose tumors had EGFR-activating mutations. In addition, response rate and PFS did not differ in patients with EGFR-activating mutations, regardless of whether they received gefitinib or erlotinib. These findings are compatible with cell line findings of hypersensitive cell lines with EGFR-activating mutations responding well to both agents $(26,27)$. Multivariate analysis also revealed that having, or not having, an EGFR-activating mutation is a significant prognostic factor for PFS, while the type of EGFR-TKI used is not a significant prognostic factor.

Our previous findings of a similar response rate, better disease control rate, longer PFS and overall survival, in a multi-center, retrospective analysis of NSCLC patients with unknown tumor EGFR mutation status, were again partially documented in the present study. There was an improved response rate and longer PFS in patients without tumor EGFR-activating mutations (17). This effectiveness in patients without EGFR-activating mutations is supported by cell line findings and is also possibly due to the higher dose intensity of erlotinib than gefitinib.

Since the EGFR-activating mutation rate was high, up to $67.6 \%$ ( 73 out of 108 ), in the male patients in our study and in $61.3 \%$ (38 out of 62) of patients who were smokers, and both clinical characteristics were considered to be poor clinical predictors for EGFR-activating mutations, all of our Taiwanese or Chinese adenocarcinoma patients should receive tumor EGFR mutation analysis.

This study had several limitations, such as the retrospective study design with its inherent potential for bias, and the fact that no toxicity profiles were reported. It was considered that there may be some differences in the frequency of adverse effects between the two agents due to varying dose intensities.

In conclusion, there was no difference in treatment response rate when one or the other agent was administered to patients with tumors either with or without EGFR-activating mutations. PFS also did not differ with either agent in patients whose tumors had EGFR-activating mutations, while PFS was significantly longer for patients receiving erlotinib treatment whose tumors did not have EGFR-activating mutations. Thus, both agents may be used in patients whose tumors have EGFRactivating mutations with similar efficacy, but not in patients whose tumors do not have EGFR-activating mutations; for these, the treatment should be erlotinib.

\section{Acknowledgements}

This research was supported in part by grants from the National Science Council of the Republic of China, grant number NSC99-2314-B-075-035-MY3, Department of Health of the
Republic of China, grant number DOH100-TD-C-111-007, and Taipei Veterans General Hospital, grant number V99C1-050.

\section{References}

1. Parkin DM, Bray F, Ferlay J, et al: Global cancer statistics. CA Cancer J Clin 55: 74-108, 2005.

2. Thatcher N, Chang A, Parikh P, et al: Gefitinib plus best supportive care in previously treated patients with refractory advanced non-small-cell lung cancer: results from a randomised, placebo-controlled, multicentre study (Iressa Survival Evaluation in Lung Cancer). Lancet 366: 1527-1537, 2005.

3. Shepherd FA, Rodrigues PJ, Ciuleanu T, et al: Erlotinib in previously treated non-small-cell lung cancer. N Engl J Med 353: 123-132, 2005.

4. Chang A, Parikh P, Thongprasert S, et al: Gefitinib (IRESSA) in patients of Asian origin with refractory advanced non-small cell lung cancer: Subset analysis from the ISEL study. J Thorac Oncol 1: 847-855, 2006.

5. Kim ES, Hirsch V, Mok T, et al: Gefitinib versus docetaxel in previously treated non-small-cell lung cancer (INTEREST): a randomised phase III trial. Lancet 372: 1809-1818, 2008.

6. Mok TS, Wu Y-L, Thongprasert S, et al: Gefitinib or carboplatin-paclitaxel in pulmonary adenocarcinoma. N Engl J Med 361: 947-957, 2009.

7. Rosell R, Moran T, Queralt C, et al: Screening for epidermal growth factor receptor mutations in lung cancer. N Engl J Med 361: 958-967, 2009.

8. Zhou C, Wu YL,Chen G, et al: Efficacy results from the randomized phase III OPTIMAL (CTONG 0802) study comparing first-line erlotinib versus carboplatin (CBDCA) plus gemcitabine (GEM), in Chinese advanced non-small-cell lung cancer (NSCLC) patients (PTS) with EGFR activating mutations. Ann Oncol 21 (Suppl 8): LBA13, 2010.

9. Chen YM, Whang-Peng J and Chen CM: Review of first-line systemic therapy for metastatic non-small cell lung cancer. J Exp Clin Med 3: 116-120, 2011.

10. Janne PA and Johnson BE: Effect of epidermal growth factor receptor tyrosine kinase domain mutations on the outcome of patients with non-small cell lung cancer treated with epidermal growth factor receptor tyrosine kinase inhibitors. Clin Cancer Res 12 (Suppl 14): 4416-4420, 2006.

11. Kosaka T, Yatabe Y, Endoh H, et al: Mutations of the epidermal growth factor receptor gene in lung cancer: biological and clinical implications. Cancer Res 64: 8919-8923, 2004.

12. Shigematsu H, Lin L, Takahashi T, et al: Clinical and biological features associated with epidermal growth factor receptor gene mutations in lung cancers. J Natl Cancer Inst 97: 339-346, 2005.

13. Baselga J, Rischin D, Ranson M, et al: Phase I safety, pharmacokinetic, and pharmacodynamic trial of ZD1839, a selective oral epidermal growth factor receptor tyrosine kinase inhibitor, in patients with five selected solid tumor types. J Clin Oncol 20: 4292-4302, 2002.

14. Herbst RS, Maddox AM, Rothenberg ML, et al: Selective oral epidermal growth factor receptor tyrosine kinase inhibitor ZD1839 is generally well-tolerated and has activity in non-small-cell lung cancer and other solid tumors: results of a phase I trial. J Clin Oncol 20: 3815-3825, 2002.

15. Hidalgo M, Siu LL, Nemunaitis J, et al: Phase I and pharmacologic study of OSI-774, an epidermal growth factor receptor tyrosine kinase inhibitor, in patients with advanced solid malignancies. J Clin Oncol 19: 3267-3279, 2001.

16. Sharma SV, Bell DW, Settleman J, et al: EGFR growth factor mutations in lung cancer. Nat Rev Cancer 7: 169-181, 2007.

17. Fan WC, Yu CJ, Tsai CM, et al: Different efficacies of erlotinib and gefitinib in Taiwanese patients with advanced non-small cell lung cancer: a retrospective multi-center study. J Thor Oncol 6: 148-155, 2011.

18. Ciuleanu T, Stelmakh L, Cicenas S, et al: Erlotinib versus docetaxel or pemetrexed as second-line therapy in patients with advanced non-small-cell lung cancer (NSCLC) and poor prognosis: efficacy and safety results from the phase III TITAN study. Chicago Multidisciplinary Symposium in Thoracic Oncol: LBOA5, 2010.

19. Vamvakas L, Agelaki S, Kentepozidis NK, et al: Pemetrexed (MTA) compared with erlotinib (ERL) in pretreated patients with advanced non-small cell lung cancer (NSCLC): results of a randomized phase III Hellenic Oncology Research Group trial. Proc ASCO 28: 7519, 2010. 
20. Therasse P, Arbuck SG, Eisenhauer EA, et al: New guidelines to evaluate the response to treatment in solid tumors. European Organisation for Research and Treatment of Cancer, National Cancer Institute of the United States, National Cancer Institute of Canada. J Natl Cancer Inst 92: 205-216, 2000.

21. Lynch TJ, Bell DW, Sordella R, et al: Activating mutations in the epidermal growth factor receptor underlying responsiveness of non-small cell lung cancer to gefitinib. N Engl J Med 350: 2129-2139, 2004.

22. Paez JG, Janne PA, Lee JC, et al: EGFR mutations in lung cancer: correlation with clinical response to gefitinib therapy. Science 304: 1497-1500, 2004.

23. Moyer JD, Barbacci EG, Iwata KK, et al: Induction of apoptosis and cell cycle arrest by OSI-774, an inhibitor of epidermal growth factor receptor tyrosine kinase. Cancer Res 57: 4838-4848, 1997

24. Polack VA, Savage DM, Baker DA, et al: Inhibition of epidermal growth factor receptor-associated tyrosine phosphorylation in human carcinoma with OSI-774: dynamics of receptor inhibition in situ and antitumor effects in athymic mice. J Pharmacol Exp Ther 291: 739-748, 1999.
25. Cho BC, Im CK, Park MS, et al: Phase II study of erlotinib in advanced non-small cell lung cancer after failure of gefitinib. J Clin Oncol 25: 2528-2533, 2007.

26. Sharma SV, Bell DW, Settleman J, et al: Epidermal growth factor receptor mutations in lung cancer. Nat Rev Cancer 7: 169-181, 2007.

27. Gandhi J,Zhang J, Xie Y, et al: Alterations in genes of the EGFR signaling pathway and their relationship to EGFR tyrosine kinase inhibitor sensitivity in lung cancer cell lines. PLoS One 4: e4576, 2009.

28. Zhu CQ, Santos GC, Ding K, et al: Role of KRAS and EGFR as biomarkers of response to erlotinib in National Cancer Institute of Canada Clinical Trials Group Study BR.21. J Clin Oncol 26: 4268-4275, 2008.

29. Cappuzzo F, Ciuleanu T, Stelmakh L, et al: Erlotinib as maintenance treatment in advanced non-small cell lung cancer: a multicentre, randomised, placebo-controlled phase 3 study. Lancet Oncol 11: 521-529, 2010. 